\title{
Locating Natural Frequency Faults in HVDC Transmission Systems Based on the Rotation-Invariant Technology Signal Parameter Estimation Algorithm and the Total Least Squares Method
}

\author{
Wensi Cao $\mathbb{D}$, Xi Chen $\mathbb{D}$, and Zhaohui Li $\mathbb{1}$ \\ School of Electric Power, North China University of Water Resources and Electric Power, Zhengzhou, Henan 450011, China \\ Correspondence should be addressed to Xi Chen; 1986105725@qq.com
}

Received 18 November 2021; Revised 25 December 2021; Accepted 21 January 2022; Published 16 February 2022

Academic Editor: Trung Thang Nguyen

Copyright (c) 2022 Wensi Cao et al. This is an open access article distributed under the Creative Commons Attribution License, which permits unrestricted use, distribution, and reproduction in any medium, provided the original work is properly cited.

\begin{abstract}
After a fault occurs in a high-voltage direct current transmission (HVDC) line, the natural frequency of the fault wave contains a wealth of transient information. The mathematical relationship between the main component of the natural frequency and the fault point can be determined and used for rapid and high-precision fault locations. To accurately extract the natural frequency of a fault and improve the positioning accuracy, a single-ended ranging scheme for extracting the natural frequency of an HVDC transient traveling wave based on the rotation-invariant technology signal parameter estimation algorithm (TLS-ESPRIT) and the total least square method is proposed for the first time. The simulation platform is used to build a dual-ended unipolar and bipolar DC transmission system model, and the scheme is simulated and verified for metallic grounding at different locations and with different transition resistances at the same location. The verification experiment was carried out on the recorded wave data of the "3.21" grounding fault of the Tianguang (Tianshengqiao-Guangdong) DC line. The results showed that the algorithm can rapidly locate the ground fault in the HVDC line and provide high ranging accuracy and good practicality.
\end{abstract}

\section{Introduction}

High-voltage direct current transmission occurs in regional trunk line interconnection systems, and safe and stable operation promotes the reliability of the regional power grid and enhances the economic benefits of local power [1-4]. HVDC transmission lines pass through mountainous areas, rivers, the Gobi Desert, and sea areas. The climate and environmental conditions are severe in many of these areas. DC transmission lines are the parts of DC systems that are most prone to failure. Common faults include single-pole faults, bipole short-circuit faults, and single-level faults. Identifying the faults that most frequently occur [5-7], quickly remotely locating the fault, and eliminating the fault to ensure the reliability of the power supply are particularly important tasks. The HVDC fault location scheme mainly uses two classification standards based on the distance measurement principle and the source of fault information extraction. The former encompasses methods such as the traveling wave method, fault analysis method, and natural frequency method. The latter includes the single-ended method and the double-ended method. The traveling wave method mainly relies on the traveling wave arrival time difference $\Delta t$ for a fault between different measurement points, and this information is combined with the propagation speed $\mathrm{v}$ to determine the fault distance. The most commonly used method is wavelet transform fault location, but the accuracy of location determination is influenced by the sampling accuracy. Because the physical boundary formed by the smoothing reactor and the DC filter in a DC system can highly vary in terms of the reflection characteristics of traveling waves of different frequencies, wave head identification, and time calibration can be difficult, especially for long-distance high-impedance ground faults. The existing wave head identification technologies have not solved this fundamental problem. Therefore, there are 
unavoidable systematic errors and inherent disadvantages in distance measurements based on the traveling wave method in DC systems. The fault analysis method is based on fault record data and system parameters, which are used to construct a fault distance equation set and solve for unknown quantities through numerical analysis. Notably, the impedance method is most commonly used, and the loop impedance is calculated by measuring the single-ended fault voltage and current to perform distance measurement and positioning tasks. The accuracy depends on an accurate line model and parameter information. However, in practical applications, DC systems have complex structures and diverse operating conditions, making it difficult to accurately construct a system operating model. In addition, the impedance method is particularly susceptible to the effects of the transition resistance at the fault point, the impedance of the opposite system, and the load current. Moreover, because algorithms are often based on one or several assumptions, and these assumptions are often inconsistent with the actual conditions, the single-ended impedance method has a principal error that cannot be eliminated. The main component of the traveling wave natural frequency is used to locate faults, and there is no need to identify traveling wave heads. Only the fault waves of electrical signals within a period of time after the occurrence of a short-circuit must be extracted for spectrum analysis. Compared with AC transmission systems, DC transmission lines provide less interference information regarding fault components. It is easy to extract the principal components of natural frequencies, thus overcoming the inherent shortcomings of traveling wave head calibration and identification and the poor adaptability of the traditional faulty analysis approach to changes in the operating state. This positioning principle yields significant advantages in DC transmission fault location. The accuracy of the natural frequency range depends on whether the principal component of the traveling wave fixed frequency is accurately extracted. Early researchers mainly used traditional and mature spectrum signal analysis algorithms and used Fast Fourier Transform (FFT) to extract the main components of the solid frequency. However, the FFT's spectrum leakage, spectrum aliasing, and fence effect phenomena can only handle the overall transient electrical signal. The frequency spectrum is distributed and the localized frequency points cannot be accurately located; In view of the shortcomings of FFT, the researchers proposed continuous wavelet transform (CWT) to continue the shortterm localization and other advantages, and established a signal time-frequency processing scheme for multifrequency analysis with dynamic "time-frequency" windows. However, it is only effective for transient electrical signals in the frequency range of the known noise, and it is less effective for the white noise that exists widely in practical applications; At present, the most widely used is the use of multiple signal component algorithm (Music algorithm) to identify the natural frequency of traveling waves [8-11], but the Music algorithm needs to search for spectral peaks in the entire frequency domain, which has disadvantages such as weak real-time performance and high computational complexity. It is not conducive to the rapid processing of DC fault lines.
Recently, related researchers have put forward different proposals. For example, Reference [12, 13] proposed the Prony algorithm to extract the principal components of natural frequencies; that is, a linear combination of a set of exponential terms is used to fit equally spaced sampled data. However, because the Prony model is numerically ill-conditioned, nonstrict and optimal algorithms are very sensitive to the effects of noise. Reference [14] proposes to use variational modal decomposition (VMD algorithm) to analyze the frequency spectrum of the fault traveling wave component, but the boundary effect, the overlap of the spectrum in the global range, and the number of modes will all affect the positioning accuracy. For the HVDC line fault signal processing, it is necessary to meet the rapidity and overcome the drawbacks of the currently applied algorithms. At present, there has not yet been found a special natural frequency principal component solution for HVDC faults.

HVDC fault traveling wave belongs to the superposition of sinusoidal signal and noise signal. First, the rotationinvariant technology signal parameter estimation algorithm (Esprit algorithm) is most suitable for line spectrum analysis. The noise is realized by decomposing the fault traveling wave into signal subspace and noise subspace. The effective detection of the lower sinusoidal signal overcomes the shortcomings of the Prony algorithm's poor antinoise ability and has a higher estimation accuracy. Secondly, compared with the shortcomings of the VMD algorithm, the Esprit algorithm avoids the existence of its shortcomings in principle, and at the same time, through the high-precision identification of the signal and noise subspace, the identification accuracy of the principal components of the natural frequency is guaranteed. Finally, the Esprit algorithm will directly solve the estimated signal frequency, effectively reducing the number of calculations and calculation time, overcoming the shortcomings of the slow calculation speed of the Music algorithm, and ensuring the rapidity of fault removal.

Combining the advantages and disadvantages of the current Esprit algorithm, this paper proposes a method that combines FFT with the rotation-invariant technology signal parameter estimation algorithm (TLS-Esprit algorithm) of the total least square method and applied to the HVDC traveling wave natural frequency ranging method. First, compared with the Esprit algorithm, the TLS-Esprit algorithm has higher calculation accuracy and stability and is suitable for steady-state and transient signal processing. In addition, due to the sinusoidal performance of the DC transient signal, FFT can quickly and accurately determine the signal order and use its recognition result as an input of Esprit, which improves the current observation and estimation method used to determine the signal order caused by the TLS-Esprit algorithm error, so the estimation accuracy is further improved. Finally, through the comparison of simulation calculations, the correctness and feasibility of the TLS-ESPRIT algorithm HVDC fault location scheme and the engineering practicability are verified, which fills the gap in the research of the main component identification algorithm of the natural frequency of the HVDC fault signal. 


\section{Natural Frequency Generation Mechanism and Ranging Principle}

Since high-voltage HVDC transmission lines are used for large-capacity long-distance transmission, a single-pole shortcircuit fault occurs in the DC transmission line area. The control systems of the converter stations on both sides are located at longer electrical distances than the fault point, thus influencing the fault signal. Small values can be ignored, so the transient signal on the line after the fault can be divided into a normal component and a faulty component, which are superimposed. As shown in the equivalent model diagram of DC system fault occurrence in Figure 1, the additional fault component at the fault point will spread to both sides, and multiple reflections will occur on the rectifier side, on the inverter side, and at the fault point. A series of electrical signal fault traveling waves will be captured, and this set is regarded as a series of different frequency harmonics that are superimposed. The frequency corresponding to this series of harmonics is called the natural frequency, and the low-order and largest amplitude harmonics corresponding to the frequency are the main components of the natural frequency [15]; moreover, the high-frequency component decreases in amplitude as the frequency increases.

When the traveling wave encounters an open point, the polarity of the reflected wave remains unchanged; when the traveling wave encounters a short-circuit point, it undergoes negative total reflection and the wave polarity changes. Suppose the traveling wave velocity is $v$ and that the traveling wave starts at a short-circuit point and propagates to both sides. If the rectifier side is in an ideal open state, the traveling wave will undergo positive total reflection and return to the short-circuit point, and then at the short-circuit point (assuming metal grounding at the short-circuit point), negative total reflection occurs, resulting in flow to the rectifier side. The formula for the propagation period $T_{1}$ of the fault traveling wave is as follows:

$$
T_{1}=\frac{4 d_{1}}{v} \text {. }
$$

If the fault traveling wave reaches the rectifier side when the rectifier side is short circuited, negative total reflection occurs again, and then negative total reflection occurs again at the short-circuit point. The formula for the traveling wave propagation period $T_{2}$ is as follows:

$$
T_{2}=\frac{2 d_{1}}{v}
$$

If there is a transition resistance at the short-circuit point, the traveling wave reflection will not be consistent with the above ideal situation. The reflection conditions are related to the actual parameters of the DC transmission system and the short-circuit conditions, but a mathematical model can be used to simulate such conditions. The reflection angles on both sides of the converter station and the short-circuit point can be calculated, and the propagation coefficient of the DC transmission line under actual conditions can be determined; consequently, the mathematical connection between the fault location and the fault traveling wave can be established.

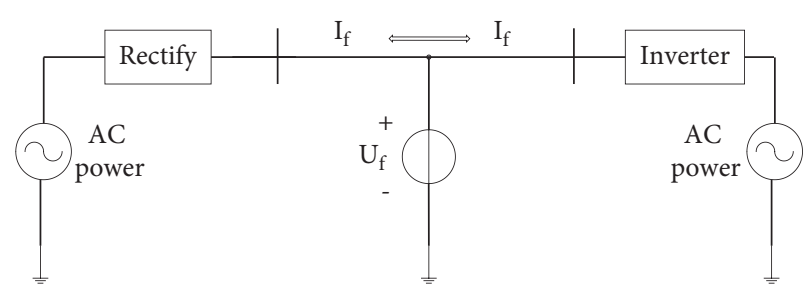

Figure 1: Failure model diagram.

The research focus of this study involves the relationship between the DC natural frequency and fault distance. HVDC transmission lines and boundary conditions must be equivalent. For unipolar DC systems, the input-state-output model can be established with reference to single-phase AC lines [15], as shown in Figure 2. Notably, the equivalent Thevenin model of a transmission line and its terminal system is illustrated.

In Figure 2, $Z_{1}$ is the equivalent impedance of the rectifier side; $Z_{2}$ is the characteristic impedance of the transmission line; $R$ is the ground resistance; $U$ is the equivalent power supply of the rectifier system; $S_{1}$ and $S_{2}$ are state variables.

$$
\begin{aligned}
& \beta_{1}(s)=\frac{Z_{1}-Z_{2}}{Z_{1}+Z_{2}}, \\
& \beta_{2}(s)=\frac{R-Z_{2}}{R+Z_{2}} .
\end{aligned}
$$

In equations (3) and (4), $S_{1}$ is the Laplace transform of the reflection coefficient on the rectifier side and $S_{2}$ is the Laplace transform of the reflection coefficient of the fault point.

$$
T(s)=e^{-s \tau} \text {. }
$$

In formula (5), $T(s)$ is the Laplace delay operator.

$$
1-T(s) \beta_{1}(s) T(s) \beta_{1}(s)=0 .
$$

The solution to equation (6) is the root of the natural frequency.

$$
\begin{aligned}
& \theta_{1}=\arccos \frac{\operatorname{Re}\left(\beta_{1}\right)}{\left|\beta_{1}\right|}, \\
& \theta_{2}=\arccos \frac{\operatorname{Re}\left(\beta_{2}\right)}{|\beta 2|} .
\end{aligned}
$$

In formulae (7) and (8), $\theta_{1}$ is the fault traveling wave reflection angle on the rectifier side and $\theta_{2}$ is the fault traveling wave reflection angle at the fault point.

$$
f=\left\{\begin{array}{l}
\frac{v}{4 \pi L}\left(\theta_{1}+\theta_{2}+\pi+2 k \pi\right), \\
\left(0 \leq \theta_{1}+\theta_{2} \leq \pi\right), \\
\frac{v}{4 \pi L}\left(\theta_{1}+\theta_{2}+2 k \pi\right), \\
\left(\pi \leq \theta_{1}+\theta_{2} \leq 2 \pi\right),
\end{array}\right.
$$




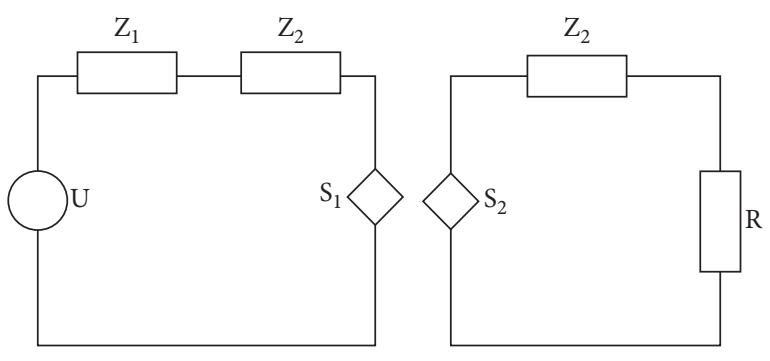

FIgURE 2: Thevenin equivalent circuit diagram.

Equation (9) is the natural frequency of the fault traveling wave obtained in the frequency domain.

$$
d=\left\{\begin{array}{l}
\frac{(\theta 1+\theta 2) \times v}{2 \omega}, \\
\operatorname{Re}(\beta 1 \times \beta 2)>0, \\
\frac{(\theta 1+\theta 2-\pi) \times v}{2 \omega}, \\
\operatorname{Re}(\beta 1 \times \beta 2)<0,
\end{array}\right.
$$

Equation (10) is the formula for the distance to the fault and $v$ is the traveling wave velocity corresponding to the natural frequency.

$$
\begin{aligned}
& v=\frac{c \omega}{\sqrt{\omega^{2}+(1 / 2)\left(\left(r_{1} / l_{1}\right)-\left(g_{1} / c_{1}\right)\right)^{2}}}, \\
& \omega=2 \pi f .
\end{aligned}
$$

Formula (11) is the traveling wave velocity corresponding to the natural frequency; $c$ is the propagation speed of light in a vacuum, where $c=3 \times 10^{5} \mathrm{~km} \cdot \mathrm{s}^{-1} ; r_{1}$ is the unit resistance of the line; $l_{1}$ is the inductance per unit length; $g_{1}$ is the conductance per unit length; $c_{1}$ is the capacitance per unit length. Formula (12) gives the angular frequency at the natural frequency.

If the two sides are similar to an ideal short-circuit (the reflection angle is $\pi$ ), the fault distance formula can be approximated as follows:

$$
d=\frac{v}{2 f}
$$

If the two sides are approximately open (the reflection angle is 0 ), the fault distance formula can be approximated as follows:

$$
d=\frac{v}{4 f}
$$

In an actual DC transmission system, the converter station is equipped with a smoothing reactor and a DC filter, and both ends of the fault traveling wave are approximately positively reflected. However, due to interference from other factors on-site, an approximation can be obtained based on the result of formula (15). The resulting approximate formula can be multiplied by a correction coefficient $\varepsilon$.

$$
d_{j}=e \frac{v}{4 f}
$$

\section{TLS-Esprit Frequency Estimation Principle}

The signal parameter estimation method (ESPRIT) based on rotation-invariant technology is a signal frequency estimation method based on the eigendecomposition of the correlation matrix in frequency estimation with a nonparametric model [16-19]. At present, there are two classic methods based on ESPRIT: least squares (LS) and total least squares (TLS). The performance of the two algorithms is similar, but under the condition of a low signalto-noise ratio, TLS performs better [20-22]. This paper chooses to use the TLS-ESPRIT method to extract the principal components of natural frequencies.

3.1. ESPRIT Frequency Estimation. At the moment of occurrence of a DC line fault, the electrical signal recorded by the converter station can be regarded as a superposition of a series of sinusoidal signals and noise components. It is assumed that $N$ signals at time $t$ are incident for $M$ receiving element arrays with azimuth angles $\theta_{1} \ldots . \theta_{n}(N<M)$.

$$
\left\{\begin{array}{l}
x_{k}(t)=\sum_{k=1}^{N} \alpha_{k} e^{j \omega_{k} t} s_{i}(t)+v_{k}(t), \\
k=1, \ldots L \ldots, M,
\end{array}\right.
$$

In formula (16), $x_{k}(t)$ is the signal received by the kth element; $\alpha_{k}$ is the amplitude of the sine component; $\omega_{k}$ is the frequency of the sine component; $V_{k}$ is the noise component.

$$
\left\{\begin{array}{l}
\vec{X}(t)=\vec{A} \vec{S}(t)+\vec{V}(t), \\
\vec{A}=\left[\overrightarrow{\alpha_{1}}(\omega), L, \overrightarrow{\alpha_{1}}(\omega)\right]
\end{array}\right.
$$

In formula (17), $\vec{X}$ is the data matrix; $\vec{A}$ is the steering vector matrix; $\vec{S}$ is the spatial signal matrix; and $\vec{V}$ is the noise data matrix.

A random process is defined as follows:

$$
\left\{\begin{array}{l}
y(t)=x(t+1), \\
\vec{Y}(t)=\vec{A} \vec{f} \vec{S}(t+1)+\vec{V}(t+1),
\end{array}\right.
$$

In formula (18), $\vec{f}=\operatorname{diag}\left\{e^{j f_{1}}, L, e^{j f_{n}}\right\}$ and $\vec{Y}$ are data matrices, and $\vec{X}$ and $\vec{Y}$ have a phase difference of one $f_{\overrightarrow{4}}$.

The signal direction is related to the matrices $\vec{A}$ and $\vec{f}$. As long as the rotation-invariant relationship $\vec{f}$ between the two data matrices is valid, the signal arrival information can be obtained with the following formula:

$$
\theta_{k}=\arcsin \frac{f_{k} \lambda_{k}}{2 \pi \Delta}
$$

In formula (19), $\Delta$ is the distance between the two data matrices. 
The covariance matrix $Z$ and autocorrelation matrix $R_{x x}$ can now be established:

$$
\begin{aligned}
& \left\{\begin{array}{l}
R_{x x}=E\left[\vec{Z}(t), \vec{Z}^{H}(t)\right], \\
R_{x x}=\vec{B} \vec{P}_{s} \vec{B}^{H}+\vec{P}_{v},
\end{array}\right. \\
& \left\{\begin{array}{l}
Z=\left[\begin{array}{l}
\vec{X} \\
\vec{Y}
\end{array}\right]=\left[\begin{array}{c}
\vec{A} \\
\vec{A} \vec{f}
\end{array}\right] \vec{S}+\vec{V}, \\
\vec{B}=\left[\begin{array}{c}
\vec{A} \\
\vec{A} \vec{f}
\end{array}\right] .
\end{array}\right.
\end{aligned}
$$

Next, singular value decomposition (SVD) can be performed for $R_{x x}$ :

$$
R_{x x}=U_{s} \sum_{s} V_{S}^{H}+U_{n} \sum_{n} V_{n}^{H} .
$$

In formula (21), $U_{S}$ is the signal subspace corresponding to the eigenvectors of large eigenvalues and $U_{n}$ is the noise subspace corresponding to small eigenvalues.

Since the signal subspace constructed based on the large eigenvector is equal to the subspace constructed by the steering vector matrix, a nonsingular matrix $T$ that satisfies formula (22) exists:

$$
U_{s}=\vec{B} T \text {. }
$$

The two subspaces divided by the large feature vector $U_{S}$ satisfy the following relationship.

$$
\begin{aligned}
U_{s} & =\left[\begin{array}{l}
U_{s 1} \\
U_{s 2}
\end{array}\right]=\left[\begin{array}{c}
\vec{A} T \\
\vec{A} \vec{f} T
\end{array}\right], \\
U_{s 2} & =U_{s 1} T^{-1} f T=U_{s 1} \psi .
\end{aligned}
$$

If $\vec{A}$ is a full-rank matrix, then:

$$
T^{-1} \psi T=f .
$$

Substituting (24) into (19) yields the incident phase information, and a frequency estimate can be obtained.

3.2. TLS-Optimized Frequency Estimation Process. If $\Delta U_{S 1}$ and $\Delta U_{S 2}$ disturbances occur at both ends of equation (23), the objective constraint is that the disturbance norm is the smallest, and the optimal solution for $\psi$ in the matrix equation in formula (25) is calculated.

$$
U_{\mathrm{s} 2}+\Delta U_{\mathrm{s} 2}=\left(U_{\mathrm{s} 1}+\Delta U_{\mathrm{s} 1}\right) \psi
$$

Formula (24) can be mathematically transformed to obtain formula (26).

$$
\left\{\begin{array}{l}
\left(\left[-\Delta U_{s 2} \Delta U_{s 1}\right]+\left[-U_{s 2} U_{s 1}\right]\right)\left[\begin{array}{l}
1 \\
\psi
\end{array}\right]=0, \\
(\Delta U+U) Z=0 .
\end{array}\right.
$$

The TLS solution is equivalent to $\min \|\Delta U\|^{2}$ and is subject to the constraints in formula (26).
Based on the definition $U_{P}=\left[\begin{array}{ll}U_{S 1} & U_{S 2}\end{array}\right]$, there is a unitary matrix that is orthogonal to $U_{P}$ and the space formed by the submatrices of $U_{P}$. The unitary matrix can be obtained by the eigen decomposition of $U_{P}$.

$$
U_{p}^{H} U_{p}=E D E^{H} .
$$

In formula (27), $D$ is a diagonal matrix composed of eigenvalues, and $E$ is a matrix composed of $D$ corresponding eigenvectors.

$$
\begin{aligned}
E & =\left[\begin{array}{ll}
E_{11} & E_{12} \\
E_{21} & E_{22}
\end{array}\right], \\
\psi_{T L S} & =-E_{21} E_{22}^{-1} .
\end{aligned}
$$

The eigenvalue $\lambda_{k}$ of $\psi_{T L S}$ must be determined $(k=1,2,3 \ldots \ldots)$.

$$
\begin{gathered}
\omega_{k}=\frac{\arg \lambda_{k}}{T_{s}}, \\
\delta_{k}=-\frac{\ln \left|\lambda_{k}\right|}{T_{s}} .
\end{gathered}
$$

Equation (29) is the signal frequency calculation formula, and equation (30) is the signal attenuation coefficient calculation formula. $T_{S}$ is the system sampling period, and $k$ is the order of the sampling model.

For an actual fault waveform, the number of sine waves is unknown (its value determines the model order $k$, which is equal to 2 times the number of sine waves). The relevant literature uses the change in the observation matrix to determine the order, but this approach is not applicable in our case. Thus, FFT is used to determine the value of $k$. The signal frequency and attenuation coefficient are further calculated with the TLS approach to obtain the amplitude and phase of each signal component. The FFT algorithm is the simplest algorithm used to process time-domain featureless signals into frequencydomain feature signals. Since a periodic signal is inversely proportional to the corresponding spectral line interval, the nonperiodic signal $T \longrightarrow \infty$, the repetition frequency $\omega \longrightarrow \infty$, the spectral line approaches 0 , and the discrete spectrum approximates a continuous spectrum [23, 24].

$$
\left\{\begin{array}{l}
F(\omega)=\lim _{\omega \longrightarrow 0} \frac{2 \pi F(n \omega)}{\omega}, \\
F(\omega)=\lim _{T \longrightarrow 0} F(n \omega) T .
\end{array}\right.
$$

The Fourier transform of a nonperiodic continuous-time signal $f(t)$ can be expressed as follows:

$$
F(\omega)=\int_{-\infty}^{\infty} f(t) e^{-j \omega t} \mathrm{~d} t .
$$

In formula (32), $F(\omega)$ is called the spectral density function of the original function $f(t)$, which is referred to as the spectral function. The number of sine waves can be determined through a spectral function graph, and the model order $k$ can be obtained. 


$$
\left\{\begin{array}{l}
m_{k}=2\left|\alpha_{k} e^{j \omega_{k} t}\right| \\
\phi_{k}=j \omega_{k} t
\end{array}\right.
$$

In formula (33), $m_{k}$ is the amplitude of the corresponding signal component, and $\phi_{k}$ is the phase of the corresponding signal component.

The following process is the principal component process used to determine the spectral estimate of the natural frequency with TLS-ESPRIT.

(1) Input the fault signal, and construct the matrices $X(t)$ and $Y(t)$

(2) Construct covariance matrix $Z$ and autocorrelation matrix $R_{X X}$

(3) Perform SVD decomposition on the autocorrelation matrix $R_{X X}$ to obtain the corresponding signal subspace $U_{s}$

(4) Define the matrix $U_{P}=\left[\begin{array}{ll}U_{S 1} & U_{S 2}\end{array}\right]$ by constructing $U_{s}$ to obtain the calculated eigenvector matrix $E$

(5) Calculate $\psi_{T L S}$ and obtain the component amplitude and phase of the corresponding signal

(6) Determine the principal components of the natural frequency

\section{Bipolar Line Signal Decoupling}

For unipolar transmission lines, the coupling degree between the transient signal and the ground is low, and mutual inductance can be ignored. However, due to the strong coupling relationship between the positive and negative poles of the bipolar power transmission system, there is mutual interference between transient information. It is necessary to decouple the original transient signal. Traditional transformation methods [25-27] include the symmetric component method, Karenbauer transformation, Kedpohl transformation, and Clark transformation. The Karenbauer transformation is the most commonly used decoupling method for DC transmission systems. This paper uses the Karenbauer transformation principle to derive the Karenbauer modulus transformation matrix for a DC transmission line. The DC cable is equivalent to a uniform line. The mathematical matrix of bipolar electrical signals is a second-order balanced matrix.

$$
p=\left[\begin{array}{cc}
p_{s} & p_{m} \\
p_{m} & p_{s}
\end{array}\right]
$$

In formula (34), $p_{s}$ is a matrix of self-inductance coefficients, and $p_{m}$ is a matrix of mutual inductance coefficients.

The eigenvalues $\lambda_{1}$ and $\lambda_{2}$ of the matrix $p$ must be determined.

$$
\left\{\begin{array}{l}
\lambda_{1}=p_{m}+p_{s} \\
\lambda_{2}=-p_{m}+p_{s}
\end{array}\right.
$$

Correspondingly, the eigenvectors $x_{1}$ and $x_{2}$ must be obtained.
TABLE 1: Unipolar HVDC parameters.

\begin{tabular}{lc}
\hline Physical quantity & Parameter \\
\hline Resistance & $0.015 \Omega \cdot \mathrm{km}^{-1}$ \\
Inductance & $0.792 \mathrm{mH} \cdot \mathrm{km}^{-1}$ \\
Capacitance & $14.4 \mathrm{nF} \cdot \mathrm{km}^{-1}$ \\
Smoothing reactor inductance & $0.5 \mathrm{H}$ \\
AC filter & $50 \mathrm{~Hz} ; 40 \mathrm{Mvar}$ \\
\hline
\end{tabular}

$$
\left[\begin{array}{cc}
p_{s} & p_{m} \\
p_{m} & p_{s}
\end{array}\right]\left[\begin{array}{l}
x_{1} \\
x_{2}
\end{array}\right]=\left(p_{s}+p_{m}\right)\left[\begin{array}{l}
x_{1} \\
x_{2}
\end{array}\right]
$$

Then, we solve the following:

$$
\left[\begin{array}{cc}
p_{s} & p_{m} \\
p_{m} & p_{s}
\end{array}\right]\left[\begin{array}{l}
x_{1} \\
x_{2}
\end{array}\right]=\left(p_{s}-p_{m}\right)\left[\begin{array}{l}
x_{1} \\
x_{2}
\end{array}\right]
$$

Additionally, a solution is obtained for the case of

$$
x_{1}=-x_{2} .
$$

For $x_{1}=(1 / 2)$, we can obtain the modulus transformation matrix $S$.

$$
S=\frac{1}{2}\left[\begin{array}{cc}
1 & 1 \\
1 & -1
\end{array}\right]
$$

In this paper, the transient voltage signal is used as the simulation signal, and the modulus decomposition formula is as follows:

$$
K_{m}=\left[\begin{array}{c}
U_{+} \\
U_{-}
\end{array}\right]=S^{-1}\left[\begin{array}{c}
U_{+}^{\prime} \\
U_{-}^{\prime}
\end{array}\right]
$$

In formula $(40),\left[\begin{array}{c}U_{+}^{\prime} \\ U_{-}^{\prime}\end{array}\right]$ is the recorded voltage fault matrix, and $K_{m}$ is the decoupled voltage matrix.

\section{Building the HVDC Transmission Model}

There are currently three types of HVDC transmission models: distributed parameter models, equivalent thermal circuit models, and finite element models. Equivalent thermal circuit models are mainly based on the characteristics of thermal circuits and involve layers of cable circuits, which are used to create a mathematical model. The finite element model discretizes each unit and only uses nodes to connect units. Each unit is established with a suitable interpolation function, and finally, all the equations for a unit are combined to obtain the solution of the entire equation set. In practical applications, distributed parameter models are used. With reference to the basic structure of the singlestage earth-loop DC transmission system, a $500 \mathrm{kV}, 300 \mathrm{~km}$ single-stage 12-pulse HVDC simulation model and $a$ $\pm 230 \mathrm{kV}$ flexible bipolar $100 \mathrm{~km}$ DC transmission system were built with an electrical simulation platform. The detailed system parameters are shown in Tables 1 and 2 . Figure 3 is a structure diagram of the unipolar 12-pulse DC $500 \mathrm{kV}$ system, and Figure 4 is a structure diagram of the VSC-HVDC $\pm 230 \mathrm{kV}$ system. Figure 5 below is a flow chart of DC system fault locations based on the TLS-ESPRIT 
TABLE 2: Bipolar HVDC parameters.

\begin{tabular}{lc}
\hline Physical quantity & Parameter \\
\hline Line model & PI \\
Transmission capacity & $2000 \mathrm{MW}$ \\
Smoothing reactor inductance & $8 \mathrm{mH}$ \\
AC filter & $50 \mathrm{~Hz} ; 40 \mathrm{Mvar}$ \\
\hline
\end{tabular}

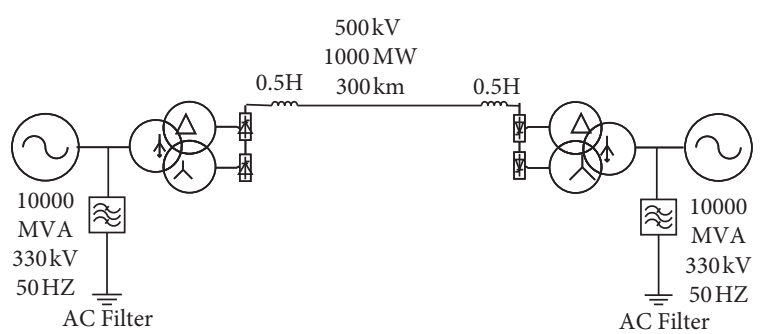

FIgURE 3: Structure diagram of the single-pole 12-pulse DC $500 \mathrm{kV}$ system.

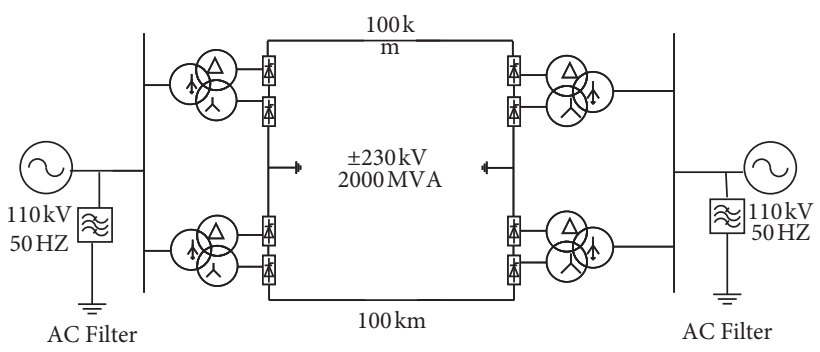

Figure 4: Structure diagram of the VSC-HVDC $\pm 230 \mathrm{kV}$ system.

algorithm. For an internal fault, the direction of the sudden change in the fault current at both ends is opposite; for an external fault, the direction of the sudden change in the current at both ends is the same. This difference is used as the basis for assessing the faults inside and outside the area. If a fault is an external fault, the output fault location is the signal outside the area; if a fault is an internal fault, the fault distance $d$ is output according to the corresponding process.

\section{Simulations and Experiments}

In actual production, both the rectifier side and the inverter side are equipped with transformers to collect signals. Therefore, the simulation uses the transmission line within $150 \mathrm{~km}$ of the rectifier side as an example (the transmission line is $300 \mathrm{~km}$, but due to symmetry, only the interval $0 \sim 150 \mathrm{~km}$ from the rectifier side is considered in this fault location research). Figure 6(a) is a simulation diagram of a $75 \mathrm{~km}$ metallic grounding voltage fault in a unipolar transmission line, and Figure $6(\mathrm{~b})$ is a simulation diagram of a $75 \mathrm{~km}$ unipolar transmission line with a grounding voltage fault with a transition resistance of 50 .

Figure 7(a) is the FFT spectrum analysis diagram of a $75 \mathrm{~km}$ unipolar transmission line with metallic grounding, and Figure 7(b) is the FFT spectrum analysis diagram of a $75 \mathrm{~km}$ unipolar transmission line grounded with a transition resistance of 50 .

Figure $8(\mathrm{a})$ is a frequency estimation diagram produced with TLS-ESPRIT for a $75 \mathrm{~km}$ metallic grounding fault voltage signal, and Figure $8(\mathrm{~b})$ is a frequency estimation diagram produced with TLS-ESPRIT for a $75 \mathrm{~km}$ unipolar transmission line grounded with a transition resistance of 50. The main component of the natural frequency can be observed from the figure, and the recognition effect is obvious and accurate.

The metallic grounding fault parameters for unipolar transmission lines, which vary at different short-circuit points, are set from 20 to $150 \mathrm{~km}$, and the fault time is varied from 0.7 to $0.85 \mathrm{~s}$. Table 3 presents the corresponding experimental simulation data.

A short-circuit is created in the unipolar transmission circuit by adjusting the transition resistance; the fault point is $75 \mathrm{~km}$, and the transition resistance is varied from 10 100. Table 4 shows the corresponding experimental simulation data.

For a metallic grounding fault point on a bipolar transmission line, the following parameters were used: short-circuit points were explored in the range of $10 \sim 50 \mathrm{~km}$. Table 5 presents the corresponding experimental simulation data.

Tables 6-8 show the result data table of distance measurement using different algorithms for the fault voltage traveling wave at the same time at the same fault point. Through comparison and analysis, it can be concluded that the improved TLS-ESPRIT algorithm has a higher accuracy of distance measurement.

The Tianguang (Tianshengqiao-Guangzhou) DC line grounding fault occurred $241.92 \mathrm{~km}$ from the Guangzhou converter station, as shown in Figure 9. The recorded voltage fault was recorded during the entire transient process at Guangzhou Station after the fault occurred [28].

TLS-ESPRIT spectrum estimation was performed in the $0.5 \mathrm{~s}$ interval after a fault occurred. As shown in Figure 10, the main component of the natural frequency was $350 \mathrm{~Hz}$. The calculated fault distance (from Guangzhou station) was $242.16 \mathrm{~km}$, the actual distance was $241.92 \mathrm{~km}$, the error was $0.24 \mathrm{~km}$, and the percent error was $0.10 \%$.

An error parameter analysis was performed as follows $[29,30]$ :

(1) Measure the data transmission error of the fault recorder during the transmission process

(2) Measure the time delay of transmission

(3) Determine the inherent error of the transformer

(4) Obtain the final calculation error 


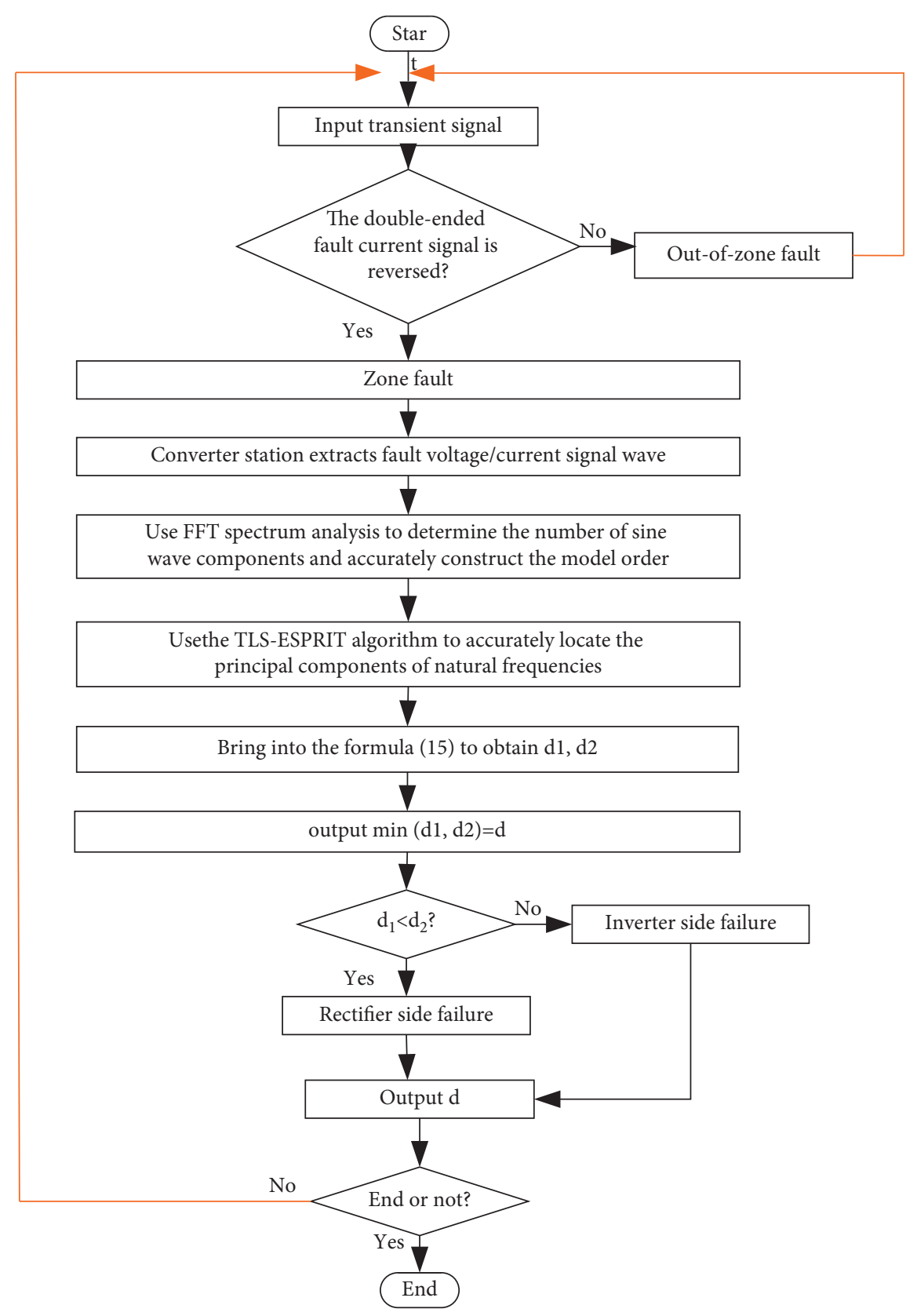

FIGURE 5: Flow chart of DC system fault locations based on the TLS-ESPRIT algorithm.

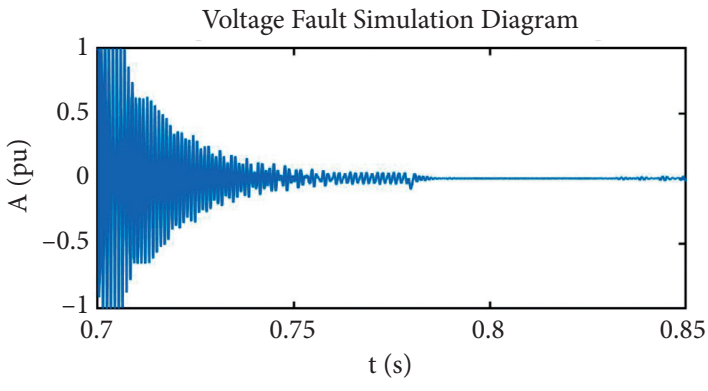

(a)

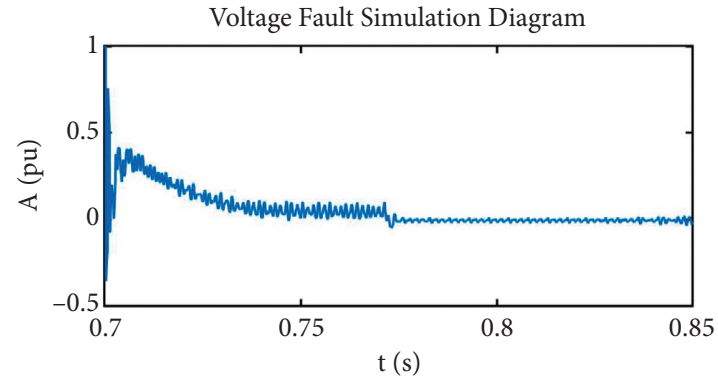

(b)

Figure 6: Voltage fault wave. (a) Metallic grounding. (b) Grounding based on transition resistance. 


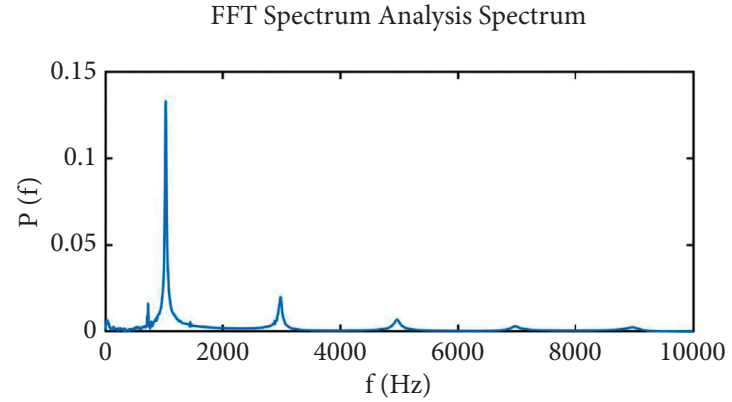

(a)

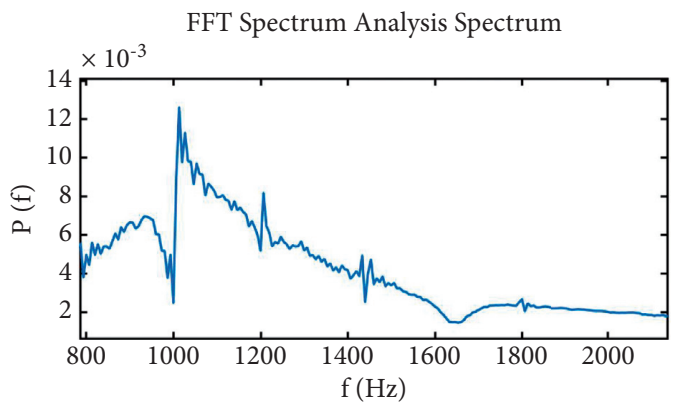

(b)

FIGURE 7: FFT spectrum analysis. (a) Metallic grounding. (b) Grounding based on transition resistance.

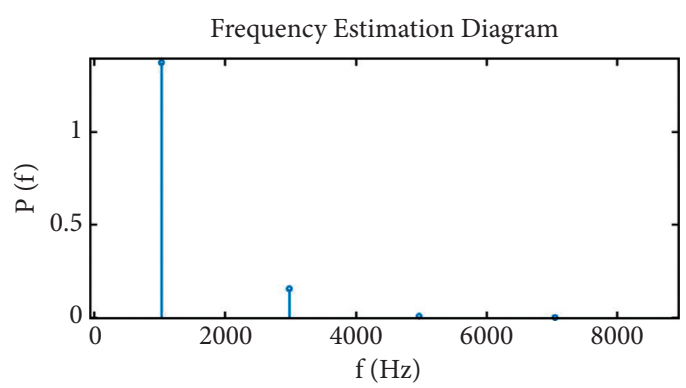

(a)

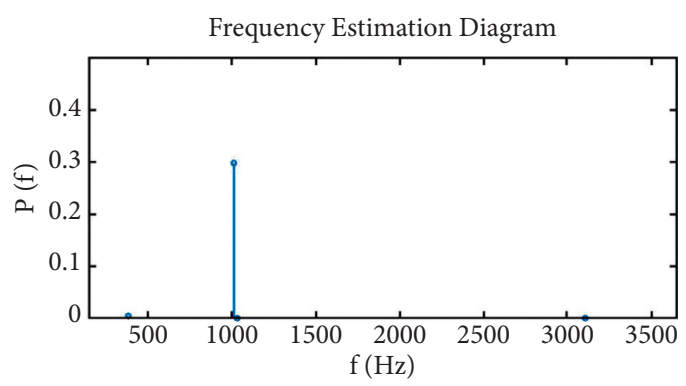

(b)

FIgURE 8: TLS-ESPRIT frequency estimation diagram. (a) Metallic grounding. (b) Grounding based on transition resistance.

TABLE 3: S-ESPRIT unipolar HVDC metallic short-circuit distance measurements.

\begin{tabular}{lccc}
\hline Actual point of failure $(\mathrm{km})$ & Natural frequency principal component $(\mathrm{Hz})$ & Calculated ranging point $(\mathrm{km})$ & Error $(\%)$ \\
\hline 20 & 3737 & 20.17 & +0.85 \\
50 & 1506 & 50.04 & -0.08 \\
75 & 1011 & 74.55 & -0.60 \\
100 & 752 & 100.23 & +0.23 \\
125 & 604 & 124.79 & -0.17 \\
150 & 502 & 150.15 \\
\hline
\end{tabular}

TABLE 4: TLS-ESPRIT short-circuit distance measurements for different transition resistance values.

\begin{tabular}{lccc}
\hline Transition resistance $(\Omega)$ & Natural frequency principal component $(\mathrm{Hz})$ & Calculated ranging point $(\mathrm{km})$ & Error $(\%)$ \\
\hline 10 & 1018 & 74.41 & -0.79 \\
20 & 1012 & 74.85 & -0.20 \\
50 & 1005 & 75.37 & -0.49 \\
100 & 995 & 75.97 & +1.30 \\
\hline
\end{tabular}

TABLE 5: TLS-ESPRIT bipolar HVDC metallic short-circuit distance measurements.

\begin{tabular}{|c|c|c|c|}
\hline Actual point of failure $(\mathrm{km})$ & Natural frequency principal component $(\mathrm{Hz})$ & Calculated ranging point $(\mathrm{km})$ & Error $(\%)$ \\
\hline 10 & 7523 & 10.02 & -0.20 \\
\hline 20 & 3795 & 19.86 & -0.70 \\
\hline 30 & 2490 & 30.27 & +0.90 \\
\hline 40 & 1870 & 40.31 & +0.78 \\
\hline 50 & 1509 & 49.95 & -0.10 \\
\hline
\end{tabular}


TABLE 6: Comparison of TLS-ESPRIT algorithm and FFT algorithm.

\begin{tabular}{lccccc}
\hline Actual point of failure $(\mathrm{km})$ & $\begin{array}{c}\text { TLS-ESPRIT } \\
(\mathrm{Hz})\end{array}$ & $\begin{array}{c}\text { Calculated ranging point } \\
(\mathrm{km})\end{array}$ & $\begin{array}{c}\text { FFT } \\
(\mathrm{Hz})\end{array}$ & $\begin{array}{c}\text { Calculated ranging point } \\
(\mathrm{km})\end{array}$ & $\begin{array}{c}\text { Increased accuracy } \\
(\%)\end{array}$ \\
\hline 20 & 3737 & 20.17 & 3877 & 19.34 & +24.50 \\
50 & 1506 & 50.04 & 1388 & 54.03 & +79.80 \\
75 & 1011 & 74.55 & 899 & 84.52 & +132.93 \\
100 & 752 & 100.23 & 733 & 102.32 & +27.50 \\
125 & 604 & 124.79 & 577 & 129.98 & +41.52 \\
150 & 502 & 150.15 & 480 & 156.25 & +40.67 \\
\hline
\end{tabular}

TABLE 7: Comparison of TLS-ESPRIT algorithm and CWT algorithm.

\begin{tabular}{lccccc}
\hline Actual point of failure $(\mathrm{km})$ & $\begin{array}{c}\text { TLS-ESPRT } \\
(\mathrm{Hz})\end{array}$ & $\begin{array}{c}\text { Calculated ranging point } \\
(\mathrm{km})\end{array}$ & $\begin{array}{c}\text { CWT } \\
(\mathrm{Hz})\end{array}$ & $\begin{array}{c}\text { Calculated ranging point } \\
(\mathrm{km})\end{array}$ & $\begin{array}{c}\text { Increased accuracy } \\
(\%)\end{array}$ \\
\hline 20 & 3737 & 20.17 & 3711 & 20.21 & +2.00 \\
50 & 1506 & 50.04 & 1508 & 49.73 & +6.20 \\
75 & 1011 & 74.55 & 1016 & 74.19 & +4.80 \\
100 & 752 & 100.23 & 755 & 99.33 & +4.40 \\
125 & 604 & 124.79 & 609 & 123.15 & +13.12 \\
150 & 502 & 150.15 & 509 & 147.35 & +16.67 \\
\hline
\end{tabular}

TABLE 8: Comparison of TLS-ESPRIT algorithm and MUSIC algorithm.

\begin{tabular}{lccccc}
\hline Actual point of failure $(\mathrm{km})$ & $\begin{array}{c}\text { TLS-ESPRIT } \\
(\mathrm{Hz})\end{array}$ & $\begin{array}{c}\text { Calculated ranging point } \\
(\mathrm{km})\end{array}$ & $\begin{array}{c}\text { Music } \\
(\mathrm{Hz})\end{array}$ & $\begin{array}{c}\text { Calculated ranging point } \\
(\mathrm{km})\end{array}$ & $\begin{array}{c}\text { Increased accuracy } \\
(\%)\end{array}$ \\
\hline 20 & 3737 & 20.17 & 3655 & 20.62 & +22.50 \\
50 & 1506 & 50.04 & 1518 & 49.65 & +12.20 \\
75 & 1011 & 74.55 & 1016 & 74.19 & +4.80 \\
100 & 752 & 100.23 & 765 & 98.53 & +12.40 \\
125 & 604 & 124.79 & 608 & 123.97 & +6.56 \\
150 & 502 & 150.15 & 507 & 148.66 & +7.93 \\
\hline
\end{tabular}

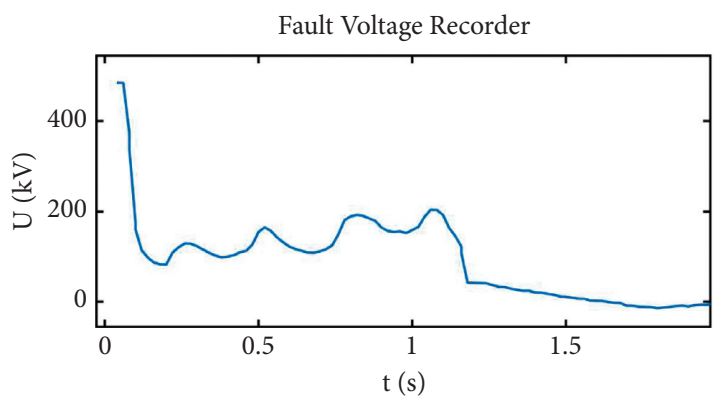

FIgURE 9: Field fault voltage recorded at Guangzhou station.

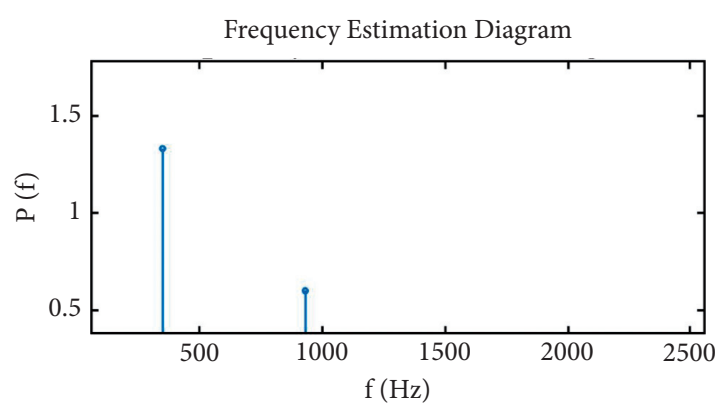

FiguRE 10: TLS-ESPRIT frequency estimation diagram.

\section{Conclusion}

(1) After simulation calculation, the proposed method of determining the traveling wave natural frequency and fault location for single-ended faults in DC transmissions based on the TLS-ESPRIT method was verified as suitable for unipolar and bipolar HVDC transmission. The different types of ground faults that occur in the lines were studied, and the results verify the theoretical correctness and feasibility of the method.

(2) The simulation results show that the fault location scheme based on TLS-ESPRIT and the TLS method yields high accuracy and low error (error within 1\%), thus meeting on-site operation and maintenance requirements.

(3) Through distance measurements and calculations with actual data for Tianguang DC faults, the proposed scheme based on the TLS-ESPRIT algorithm yields results consistent with the actual fault distance, which verifies the engineering practicality of the scheme proposed in this paper.

(4) The Music algorithm, Prony algorithm, and VMD algorithm proposed previously must search for spectral peaks in the entire frequency domain, which 
results in poor real-time performance and high computational complexity when applied to HVDC systems. The TLS-ESPRIT algorithm effectively overcomes the above shortcomings, provides accurate ranging estimates, and displays significant advantages.

\section{Data Availability}

The authors own all the data of the paper and agree to share the data after the paper is accepted.

\section{Conflicts of Interest}

The authors declare that there are no conflicts of interest regarding the publication of this paper.

\section{Acknowledgments}

This work was supported by the Henan Province Key R\&D and Promotion Special (Science and Technology) Project in 2019 (192102210229) "Research and Judgment Technology for Ground Fault Line Selection of Distribution Network Based on Big Data Clustering Analysis and FFT/STFT Algorithm" and the key scientific research project of Henan Province in 2019, "Research on Single-phase Grounding Protection of Small Resistance Grounded Distribution Network Based on the Variation of Fault Volume" (19A470006).

\section{References}

[1] H. Liu, B. Li, W. Wen et al., "Key technologies and prospects of line protection for flexible DC system," Power Grid Technology, vol. 45, no. 9, p. 15, 2021.

[2] Z. Zijun, "Pan ersheng Adhering to core technology innovation and leading the upgrading of UHVDC industry-an interview with Pan Ersheng," President of State Grid economic and Technological Research Institute China Standardization, vol. 10, no. 7, 2020.

[3] D. Cai, K. Zhou, W. Wang, H. Liu, K. Cao, and Y. Wang, "Influence of back-to-back VSC-HVDC project on the operation characteristics of Hubei power grid," Journal of Engineering, vol. 2017, no. 13, pp. 801-805, 2017.

[4] H. Liu, X. Zheng, and L. Jin, "Overview of the reliability survey of the world's large-distance and high-capacity HVDC transmission projects," High-voltage electrical appliances, vol. 38, no. 3, pp. 1-4, 2002.

[5] W. Yang, "Study on system commissioning test and project application of Lingzhou to Shaoxing UHVDC transmission project," Journal of Engineering, vol. 2017, no. 13, pp. 2188-2192, 2017.

[6] J. Yu, Z. Zhang, and X. Zheng, "A local protection and local action strategy of DC grid fault protection," Energies, vol. 13, no. 18, p. 4795, 2020.

[7] J. Xu, Y. Lu, C. Zhao, and J. Liang, "A model-based DC fault location scheme for multi-terminal mmc-hvdc systems using a simplified transmission line representation," IEEE Transactions on Power Delivery, p. 99, 2019.

[8] K. Liao, Z. He, and X. Li, "Fault location of HVDC transmission lines based on traveling wave natural frequency," Power system automation, vol. 37, no. 3, pp. 104-109, 2013.
[9] Z. Huang, "A new method of hybrid line fault location using traveling wave natural frequency," Journal of power system and automation, vol. 27, no. 11, pp. 73-79, 2015.

[10] Y. Zhang and Y. Zhu, "Fault location of multi branch transmission lines based on traveling wave natural frequency," Journal of electric power science and technology, vol. 31, no. 3, pp. 65-72, 2016.

[11] Y. Zhang, Y. Zhu, N. Zhang, M. Zhang, Y. Zheng, and $\mathrm{X}$. Huang, "Fault location of T-type transmission line based on traveling wave inherent frequency and VMD," Electrical measurement and instrumentation, vol. 54, no. 21, pp. 55-60, 2017.

[12] L. Wang, L. Huang, P. H. Huang et al., "Fault location method for DC hybrid line based on VMD-backward prediction Prony," Power Grid and Clean Energy, vol. 36, no. 10, pp. 61-67+72, 2020.

[13] M. Yang, P. Chen, Z. Yang, Y. Cao, M. T. Yang, and Chenping, "Application of Improved Prony Algorithm in single terminal fault location of transmission line," Electrotechnics and electrics, no. 5, pp. 34-38, 2014.

[14] H. Yongqi, Q. Wang, W. song, Y. Li, and X. Wang, "Fault location scheme for multi terminal DC hybrid transmission lines based on combination of natural frequency and traveling wave time domain location," Journal of power system and automation, vol. 32, no. 4, pp. 104-110, 2020.

[15] L. Wu, Single Ended Traveling Wave Fault Location Method Using Natural Frequency of Fault Traveling Wave, Southwest Jiaotong University, Cheng'du, China, 2009.

[16] X. Cai, Y. Gao, Y. Li, Y. Zhu, and W. Li, "Based on adaptive TLS-ESPRIT and improved all-phase multi-dense interspectral harmonic detection," Journal of Instruments and Instruments, vol. 41, no. 12, pp. 199-207, 2020.

[17] C. Chen, J. Tao, and G. Zheng, "Polarization smoothing and dimensionality reduction unitary ESPRIT algorithm based on MIMO radar," Signal Processing, vol. 37, no. 4, pp. 616-623, 2021.

[18] S. Zhuang, W. Zhao, D. Zhao, and S. Huang, "A Hyperharmonic dynamic analysis method based on sliding window TLS-ESPRIT algorithm," Journal of Metrology, vol. 41, no. 4, pp. 475-483, 2020.

[19] S. S. Kumar and S. Bidyadhar, "New signal subspace approach to estimate the inter-area oscillatory modes in power system using TLS-ESPRIT algorithm," IET Generation, Transmission \& Distribution, vol. 13, no. 18, pp. 4123-4140, 2019.

[20] B. Xu and Z. Zheng, "SVD filtering and TLS-ESPRIT algorithm based on stator fault characteristic detection of doublyfed induction generator," Journal of Engineering, vol. 2019, no. 18, 5196 pages, 2019.

[21] H. Zhang, Y. Wang, X. Zhang, J. Cui, and S. Yao, "Harmonic detection method and Simulation of dual adaptive neural network and fast TLS-ESPRIT," Hydroelectric Energy Science, vol. 38, no. 9, pp. 203-205+113, 2020.

[22] X. Manlin, Z. Duan, and Y. Zhenglong, “A weighted forwardbackward spatial smoothing DOA estimation algorithm based on TLS-ESPRIT: regular section," vol. E104.D, no. 6, pp. 881-884, 2021.

[23] Y. Cao, S. Tu, and S. Luwei, "Identification of electrical appliances based on FFT and genetic algorithm," Journal of Shanghai Electric Power University, vol. 36, no. 5, pp. 436-440+450, 2020.

[24] W. Wang, Research on the Application of Sparse Fast Fourier Transform [D], Chinese Academy of Surveying and Mapping, Bei'jing, China, 2018. 
[25] G. Song, L. Sen, X. Kang, D. Zhou, Z. Yang, and S. Jiale, "A new phase mode transformation matrix," Power system automation, no. 14, pp. 57-60, 2007.

[26] G. Du, Traveling Wave Protection for UHVDC Lines Based on Voltage Modulus Decomposition, Zhengzhou University, Zhengzhou, China, 2015.

[27] Z. Cheng, DC Line Traveling Wave Protection Based on Voltage Modulus Decomposition, Zhengzhou University, Zhengzhou, China, 2015.

[28] H. Jiayin, Z. Hongyang, and Y. Jiang, "Analysis and simulation of high impedance grounding fault of " 3.21 " Tianguang DC line," Guangdong Electric Power, vol. 18, no. 11, p. 4, 2005.

[29] X. Guo, F. Li, Nanbaofeng, and H. Li, "Single Super High Voltage Cable. Fault location error analysis and improvement measures for high voltage cable sheath," South China Grid Technology, vol. 15, no. 1, pp. 48-53, 2021.

[30] B. Wang and L. Yang, "Error analysis of single-end traveling wave fault location for HVDC transmission lines," Electrical applications, vol. 39, no. 10, pp. 33-38, 2020. 